\title{
Relationship between Friction Coefficient, Viscosity and Compressibility of Polyethylene Glycol
}

\author{
Kazuaki Hachiya* and Tatsuya Nitta \\ Department of Mechanical System Engineering, Okayama University of Science \\ 1-1 Ridai-cho, Okayama 700-0005, Japan \\ *Corresponding author: hachiya@mech.ous.ac.jp \\ ( Manuscript received 4 December 2007; accepted 5 March 2008; published 15 April 2008 ) \\ (Presented at JAST Tribology Conference Saga, September 2007 )
}

\begin{abstract}
An ultrasonic liquid processor with the frequency of $20 \mathrm{kHz}$ was used to cleave a principal chain in a polyethylene glycol molecule. The amount of the lubricant molecules cleaved by the ultrasonic disruption was evaluated by the kinematic viscosity measurement. As the kinematic viscosity in the water-glycol hydraulic fluids of the ethylene glycol solutions containing polyethylene glycols decreased monotonously under the condition that the ultrasound of $20 \mathrm{kHz}$ and $40 \mathrm{~W}$ was irradiated for one second every five seconds at either 20 or $40{ }^{\circ} \mathrm{C}$, the lubricant molecules could be effectively cleaved while reducing the inter-molecular polymerization. The friction coefficients, ultrasonic velocities, densities, and compressibilities in the water-glycol hydraulic fluids were not influenced by the cleavage process of the lubricant molecules. On the other hand, the kinematic viscosities in the undiluted solutions of ethylene glycol and the polyethylene glycols were proportional to their molecular weights, but the friction coefficients and compressibilities in polyethylene glycols hardly depended on their molecular weights except for those in ethylene glycol. These dependences of the frictional coefficients and compressibilities on the molecular weights resulted from a structure of the lubricant oil film formed on the metal surface.
\end{abstract}

Keywords: friction coefficient, viscosity, compressibility, polyethylene glycol, ultrasonic velocity

\section{Introduction}

A lubricant is used to reduce the friction coefficient between the sliding surfaces of a machine. However, a long machine operation causes the decrease in the viscosity of the lubricant owing to the degradation by a strong mechanical shearing force ${ }^{1,2}$. The decrease in the viscosity results in the increases in the temperature and friction coefficient on the sliding surfaces, and subsequently leads to the seizure of the machine ${ }^{3,4}$. The lubricant on the sliding surface is oxidized at high temperature. Under the lubricant film condition which was as thin as that of the hydrodynamic film, the oxidation of poly- $\alpha$-olefin on the aluminum, iron, copper, and stainless steel plates was observed with the FTIR spectrophotometer ${ }^{5}$. Besides the lubricant oxidation at the high temperature, the increase in the friction coefficient occurred due to a decrease in the film thickness that was caused by the decrease in the lubricant viscosity. The viscosity of water-glycol hydraulic fluid was usually controlled by the amount of the thickener molecules, but these molecules were cleaved by the mechanical shearing force on the sliding surface. The decrease in the lubricant viscosity had been evaluated by the ASTM ultrasonic shear stability test by using the continuous ultrasonic irradiation method with the sonic oscillator operated at $10 \mathrm{kHz}^{1,6,7)}$. However, an ultrasonic liquid processor with the frequency of $10 \mathrm{kHz}$ is no longer manufactured. The purpose of this paper is to newly develop an effective method to cleave the principal chain of the thickener molecule in the water-glycol hydraulic fluid by using the processor with the frequency of $20 \mathrm{kHz}$. The friction coefficient is dependent on the viscosity of the base oil at the constant sliding speed and normal $\operatorname{load}^{8,9)}$. Another aim is to clarify the relationship between the friction coefficient and viscosity in the lubricant containing the polyethylene glycol molecules cleaved by the ultrasonic process. In order to elucidate the structure of a lubricant film formed on the sliding metal surfaces, the relationship is also compared with that between the viscosity and the compressibility obtained by the method of the ultrasonic velocity measurement.

\section{Experimental}

Ethylene glycol and polyethylene glycols with the average molecular weights of 200, 400, 600 and 1000 (Wako Pure Chemical Industries, Ltd.) were used 
without further purifications as glycol antifreeze and thickeners in water-glycol hydraulic fluids. They are abbreviated as EG, PEG200, PEG400, PEG600 and PEG1000, respectively. Water was not added into the water-glycol hydraulic fluids, because the vaporized amount of water by the ultrasonic process was much larger than that of ethylene glycol or polyethylene glycol. $30 \mathrm{~g}$ of the water-glycol hydraulic fluid containing $85 \mathrm{wt} \%$ of ethylene glycol and $15 \mathrm{wt} \%$ of either PEG400 or PEG1000 was weighed in a $50 \mathrm{~cm}^{3}$ capacity glass beaker. It had an inner diameter of $40 \mathrm{~mm}$ and a height of $60 \mathrm{~mm}$. The high intensity ultrasonic liquid processor VCX 750 with the frequency of $20 \mathrm{kHz}$ (Sonics \& Materials, INC.) was applied to cleave the molecules of polyethylene glycol in the glass beaker which was placed in a thermostat bath and was kept constant at 20 or $40 \pm 2{ }^{\circ} \mathrm{C}$ by using the integrated temperature controller. In order to prevent the heat generated by the ultrasonic cleaving process, the ultrasound under the average pulse output of $40 \mathrm{~W}$ (i.e., the instantaneous pulse output of $45 \mathrm{~W}$ ) was irradiated in the lubricants for one second every five seconds. The average cleavage ratio of PEG400 or PEG1000 molecules as the final product of PEG200 molecules was calculated from a calibration curve which was obtained by the measurement of the kinematic viscosities in the EG solutions containing the blends with different ratios of weight percent PEG400/PEG200 or PEG1000/PEG200.

The friction coefficient in the lubricant cleaved with the ultrasonic processor was measured by using a ball-on-disk arrangement on a CSEM High Temperature Tribometer. A bearing steel ball (JIS SUJ2) of $6 \mathrm{~mm}$ diameter was installed at the bottom at the ball holder, and a $50 \mathrm{~mm}$ diameter stainless steel petri dish (JIS SUS304, AS ONE Corporation) $15 \mathrm{~mm}$ high and 0.6 $\mathrm{mm}$ thick was placed in the sample holder with a blocking ring and clasps. $2 \mathrm{~g}$ of the water-glycol hydraulic fluid was added in the petri dish in the sample holder, and the liquid temperature was controlled at 40 ${ }^{\circ} \mathrm{C}$ by the heater in the tribometer. The running-in was

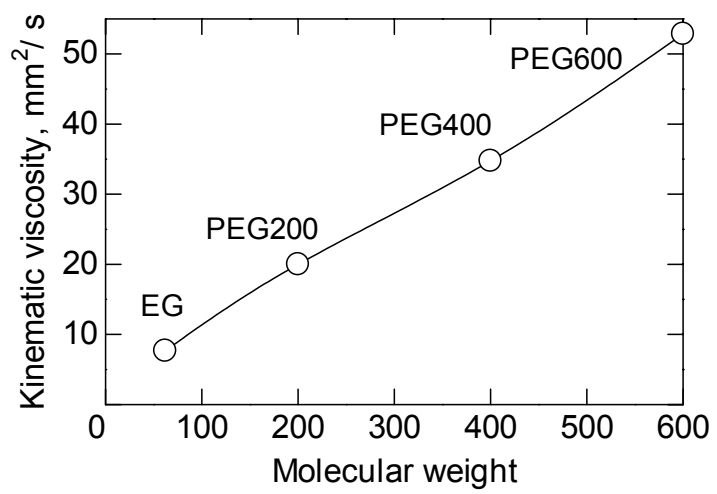

Fig. 1 Molecular weight dependence of kinematic viscosities in the undiluted solutions of EG and PEG200-PEG600 at $40{ }^{\circ} \mathrm{C}$ done in the stainless petri dish containing the lubricant for $30 \mathrm{~min}$ at $40{ }^{\circ} \mathrm{C}$, a sliding speed of $0.5 \mathrm{~cm} / \mathrm{s}$ and a constant load of $1 \mathrm{~N}$ (i.e., $0.44 \mathrm{GPa}$ ). The friction coefficient was measured for $30 \mathrm{~min}$ at sliding speeds from 0.1 to $15 \mathrm{~cm} / \mathrm{s}$, and the same temperature and load as those of the running-in.

The ultrasonic velocity in the lubricant at $40^{\circ} \mathrm{C}$ was measured by using the pulse-echo technique described by McClements and Povey ${ }^{10,11)}$. Two ultrasonic transducers with $3 \mathrm{MHz}$ frequency (PIEZOTITE 7D-10-3000B, Murata Manufacturing Co., Ltd.) were attached with adhesive to both sides of a glass cuvette (GS-40, AS ONE Corporation) $45 \mathrm{~mm}$ high, $40 \mathrm{~mm}$ wide and $1.2 \mathrm{~mm}$ thick with a $10 \mathrm{~mm}$ path length. One transducer generated the signal which traveled through the lubricant, and the other received the signal which was observed with a digital storage oscilloscope connected with the computer for the data analysis, respectively. When the pulse reached an interface between the lubricant and the glass wall, it was partly reflected and partly transmitted, and then the transducer received a series of echoes. The ultrasonic velocity $u$ was calculated within the accuracy of $0.1 \%$ from the following relationship by using the path length $d$ and the time $t$ between the first and second echoes ${ }^{10)}$ :

$$
u=\frac{2 d}{t}
$$

The adiabatic compressibility $\beta$ in the lubricant with density $\rho$ was obtained by using the formula as ${ }^{12)}$

$$
\beta=\frac{1}{\rho u^{2}}
$$

\section{Results and discussion}

Ethylene glycol and polyethylene glycols with the average molecular weights 200-600 contained in the water-glycol hydraulic fluids are a homologous series of liquid organic compounds at $40{ }^{\circ} \mathrm{C}$. Prior to the ultrasonic shear stability test with the sonic oscillator operated at $20 \mathrm{kHz}$, the molecular weight dependence on the kinematic viscosities in the undiluted solutions of EG and PEG200-600 was measured as shown in Fig. 1. Because the kinematic viscosities were proportional to the molecular weights of the lubricants, the amount of the lubricant cleaved by the ultrasonic disruption could be estimated from the decreased amount of the kinematic viscosity ${ }^{13)}$.

A continuous ultrasonic process similar to that by the ASTM method ${ }^{1,6,7)}$ was applied to cleave the principal chains of the lubricant molecules in the undiluted solution of PEG400 by using the sonic oscillator of the $100 \mathrm{~W}$ output at the frequency of 20 $\mathrm{kHz}$. Figure 2 shows the plot of the kinematic viscosity of the lubricant vs. the ultrasonic irradiation time. Although the kinematic viscosity decreased by $2 \%$ after $1 \mathrm{hr}$, it increased afterwards and became almost constant 


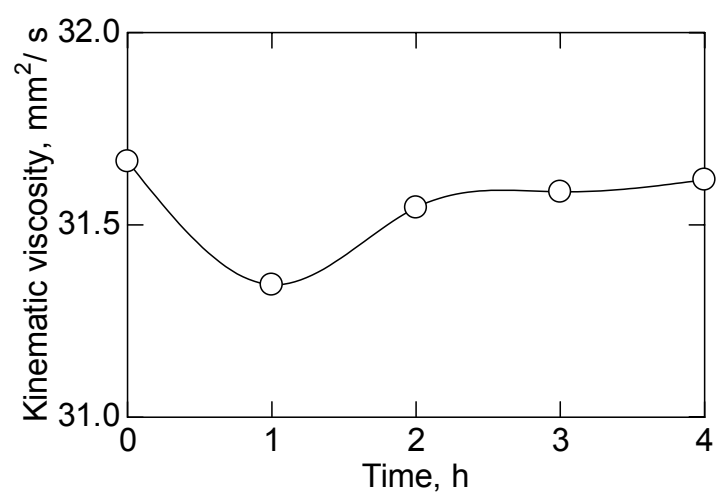

Fig. 2 Dependence of kinematic viscosity in PEG400 solution on ultrasonic irradiation time by means of the continuous irradiation of the $100 \mathrm{~W}$ output power at frequency of $20 \mathrm{kHz}$

after $2 \mathrm{hr}$. Since the initial temperature $40{ }^{\circ} \mathrm{C}$ in the lubricant reached $90{ }^{\circ} \mathrm{C}$ at the end of the ultrasonic treatment in Fig. 2, the increase in the kinematic viscosity of the lubricant seemed to be caused by the inter-molecular polymerization which was the reverse reaction towards the cleavage of the principal chain of polyethylene glycol molecule and was generated by the high ultrasonic energy at the high temperature ${ }^{14)}$. This indicated that the continuous ultrasonic irradiation at 20 $\mathrm{kHz}$ was inadequate for the effective cleaving procedure of the lubricant molecules. In order to prevent the inter-molecular polymerization caused by the increase in the lubricant temperature, the output power of the ultrasonic processor was decreased from $100 \mathrm{~W}$ to $40 \mathrm{~W}$ and the ultrasound was irradiated intermittently in the lubricants for one second every five seconds. Figure 3 shows the plots of the kinematic viscosities of the lubricants vs. the ultrasonic irradiation times under the

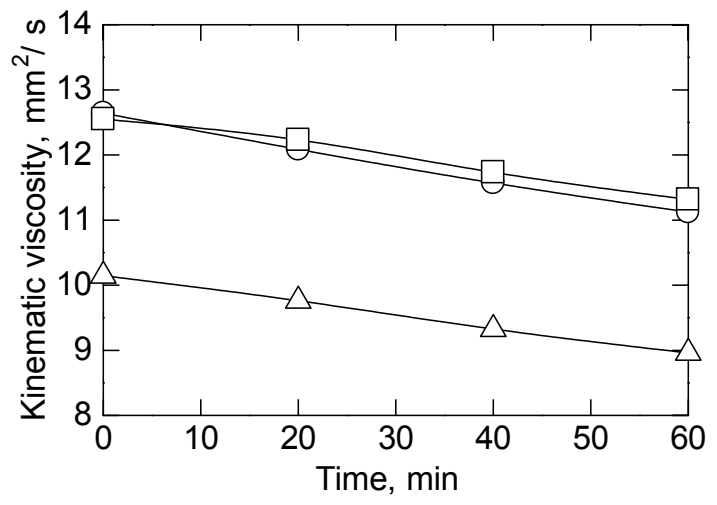

Fig. 3 Dependence of kinematic viscosities on ultrasonic irradiation times under intermittent ultrasonic irradiation: EG solution containing $15 \mathrm{wt} \%$ of PEG400 at $40{ }^{\circ} \mathrm{C}(\triangle)$, EG solution containing $15 \mathrm{wt} \%$ of PEG1000 at $20(\bigcirc)$ and $40(\square)$ ${ }^{\circ} \mathrm{C}$

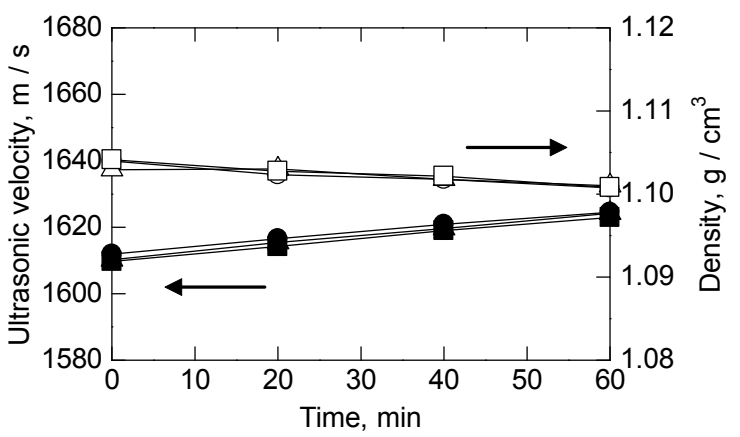

Fig. 4 Plots of ultrasonic velocities and densities in EG solution containing $15 \mathrm{wt} \%$ of PEG400 at $40{ }^{\circ} \mathrm{C}(\boldsymbol{\Delta}, \triangle)$ and EG solution containing 15 wt $\%$ of PEG1000 at $20(\bigcirc, \bigcirc)$ and 40 ( $\square, \square){ }^{\circ} \mathrm{C}$ against ultrasonic irradiation times under intermittent ultrasonic irradiation

intermittent ultrasonic irradiation at 20 and $40 \pm 2{ }^{\circ} \mathrm{C}$. The kinematic viscosities in the EG solutions containing PEG400 and PEG1000 decreased monotonously with the irradiation times at 20 and $40{ }^{\circ} \mathrm{C}$, respectively. This figure shows that the lubricant molecules could be cleaved by the intermittent ultrasonic irradiation at 20 $\mathrm{kHz}$ as seen from Fig. 1. The lowering rates of the kinematic viscosities in the lubricant containing PEG1000 were 12.0 and $9.8 \%$ at 20 and $40{ }^{\circ} \mathrm{C}$, and corresponded to the average cleavage ratios of 35 and $27 \%$, respectively. Since the lowering rate of kinematic viscosity at $20{ }^{\circ} \mathrm{C}$ was larger than that at $40{ }^{\circ} \mathrm{C}$, the cleaving process of the lubricant molecules seemed to be effective at the lower temperature than $40{ }^{\circ} \mathrm{C}$. On the other hand, the lowering rate of the kinematic viscosities in the lubricant containing PEG400 at $40{ }^{\circ} \mathrm{C}$ was $11.7 \%$, and corresponded to the average cleavage ratio of $83 \%$. The lowering rate in the lubricant containing PEG400 was larger than that in the lubricant containing PEG1000 at the same temperature. Because the kinematic viscosity in the lubricant with the large molecular weight was larger than that in the lubricant with the small molecular weight, the amount of the probe vibration per a constant frequency energy provided from the ultrasonic power supply was reduced by the frictional heat generated by the prove movement in the high viscous lubricant. As a result, the smaller lowering rate of the kinematic viscosity in PEG1000 could be attributed to the energy loss caused by the frictional work in the more viscous lubricant.

In order to obtain the compressibilities of the lubricants, the dependencies of the ultrasonic velocities and densities on the ultrasonic irradiation times were measured in the water-glycol hydraulic fluids as shown in Fig. 4. Both the ultrasonic velocities in the lubricants containing PEG400 and PEG1000 increased by $0.8 \%$ after $1 \mathrm{hr}$ at 20 and $40{ }^{\circ} \mathrm{C}$, but the densities of their lubricants decreased by $0.2 \%$ after $1 \mathrm{hr}$ at the same temperatures. Since the ultrasonic velocities and 


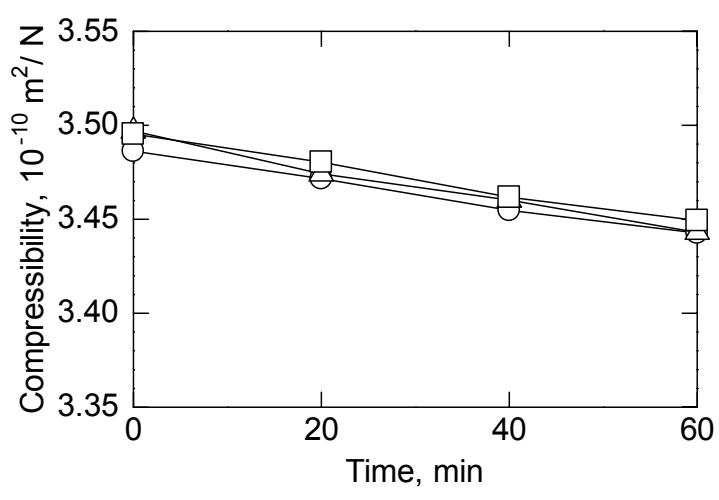

Fig. 5 Dependence of compressibilities on ultrasonic irradiation times under intermittent ultrasonic irradiation: EG solution containing $15 \mathrm{wt} \%$ of PEG400 at $40{ }^{\circ} \mathrm{C} \quad(\triangle)$, EG solution containing $15 \mathrm{wt} \%$ of PEG1000 at $20(\bigcirc)$ and $40(\square){ }^{\circ} \mathrm{C}$

densities stayed almost constant after $1 \mathrm{hr}$ in Fig. 4, they were scarcely affected by the ultrasonic cleaving processes of the lubricant molecules, when compared with the large decreases in the kinematic viscosities in Fig. 3. The compressibilities in the lubricants were calculated by using the ultrasonic velocities and densities in Fig 4, and are shown in Fig. 5. The compressibility in the lubricant containing PEG400 at $40{ }^{\circ} \mathrm{C}$ and those in the lubricant containing PEG1000 at 20 and $40{ }^{\circ} \mathrm{C}$ decreased by 1.5 and $1.3 \%$ after $1 \mathrm{hr}$, respectively.

The amount of the lubricant cleaved by the ultrasonic disruption could be evaluated from the decreased amount of the kinematic viscosity as seen from Fig. 1. As a result, it is appropriate that the data of the ultrasonic velocities measured in the lubricants were plotted against the kinematic viscosities in order to explain the relation between the ultrasonic velocity and the amount of the lubricant molecules cleaved by the ultrasonic process. Figure 6 shows the relationship between the ultrasonic velocity and the kinematic

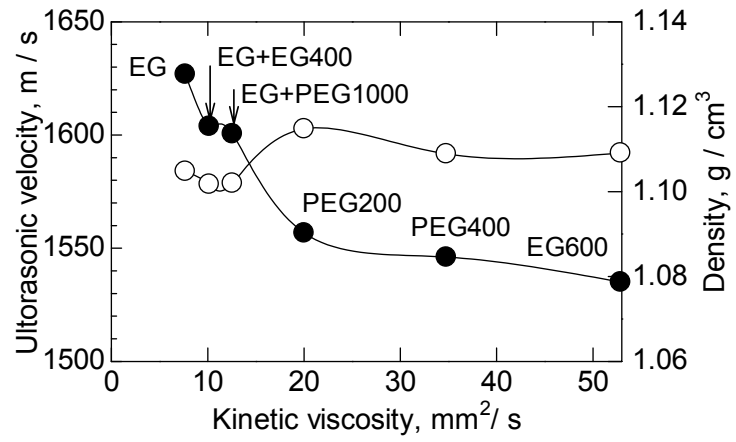

Fig. 6 Plots of ultrasonic velocity ( $O$ ) and density ( ) vs. kinematic viscosities in the lubricant at $40{ }^{\circ} \mathrm{C}$

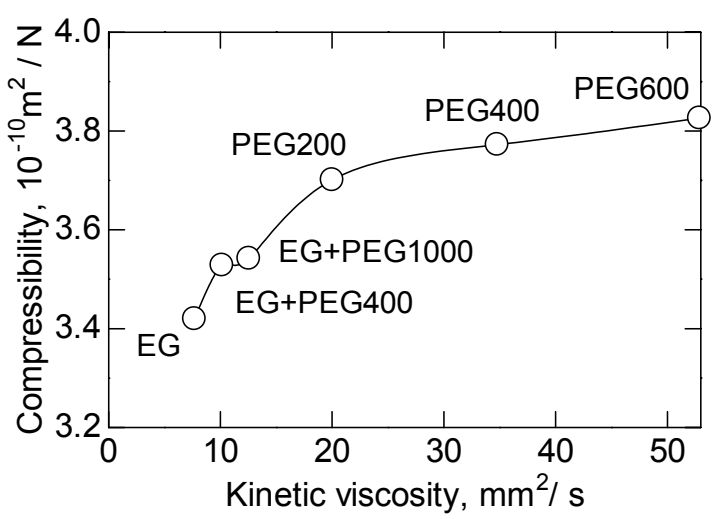

Fig. 7 Plot of compressibility vs. kinematic viscosity in the lubricant at $40{ }^{\circ} \mathrm{C}$

viscosity in the lubricant. The kinematic viscosity range, which was affected in the water-glycol hydraulic fluids of the EG solutions containing PEG400 and PEG1000 by the ultrasonic process at $40^{\circ} \mathrm{C}$, was not sufficient to investigate the detailed relationship between the ultrasonic velocity and the amount of the lubricant molecules cleaved by the ultrasonic process. As shown in Fig. 6, therefore, the ultrasonic velocities were also measured in the undiluted solutions of EG, PEG200, PEG400 and PEG600 in addition to the EG solutions containing PG400 and PEG1000 which were cleaved by the ultrasonic process for $1 \mathrm{hr}$. Since the ultrasonic velocity in the lubricant decreased when the kinematic viscosity increased, the ultrasonic velocity increased with the cleaving of the lubricant molecules. Under the same condition as the ultrasonic velocity measurement, the relationship between the density and the kinematic viscosity in the lubricant is shown in Fig. 6. The density of the lubricant was kept almost constant with increases in the kinematic viscosity, and then did not seem to be influenced by the cleaving process of the lubricant molecules. Figure 7 shows the plot of the compressibilities calculated by using the ultrasonic velocities and densities in Fig. 6 against the kinematic viscosities. The compressibilities in the lubricant increased by $10 \%$ with increases in the kinematic viscosity from EG to PEG600. The EG molecule was the minimum unit among the lubricant molecules shown in Fig. 7, and the compressibility also came to the minimum in the solution containing EG molecules. As compared in Figs. 6 and 7, the ultrasonic velocities and compresibilities in the polyethylene glycols seemed to be hardly dependent on their molecular weights except for those in ethylene glycol.

Under the same experimental conditions as the compressibility measurement at $40{ }^{\circ} \mathrm{C}$, the dependence of the friction coefficient on the kinematic viscosity was measured at a constant load of $1 \mathrm{~N}$ and sliding speeds from 0.1 to $15 \mathrm{~cm} / \mathrm{s}$ as shown in Fig. 8. The friction coefficient at a sliding speed of $0.1 \mathrm{~cm} / \mathrm{s}$ increased while the kinematic viscosity of the lubricant decreased. This 


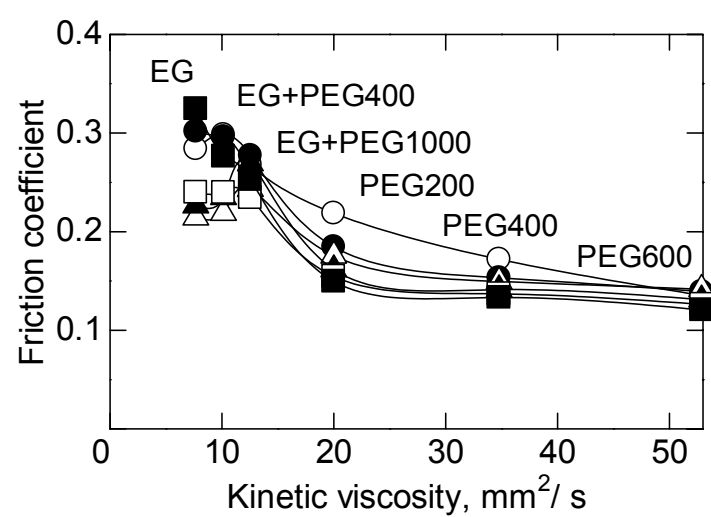

Fig. 8 Friction coefficients at a load of $1 \mathrm{~N}, 40{ }^{\circ} \mathrm{C}$ and sliding speeds of $0.1(\bigcirc), 0.5(\bigcirc)$, $1(\triangle), 5(\boldsymbol{\Delta}), 10(\square)$ and $15(\square) \mathrm{cm} / \mathrm{s}$ as a function of kinematic viscosity

indicates that the increased amount of the lubricant molecules cleaved by the ultrasonic process resulted in the increase in the friction coefficient. Since the EG and PEG molecules are the organic compounds having hydroxyl groups $-\mathrm{OH}$ at both ends of the molecules, boundary lubricating film would be formed between the functional groups on the metal oxide film of the stainless petri dishes and the EG and PEG molecules ${ }^{15)}$. Because the polar lubricant molecules with larger molecular weights bound more strongly to the metal oxide surfaces ${ }^{16}$, the increase in the friction coefficient with the decrease in the kinematic viscosity in Fig. 8 could be due to the result of the decrease in the binding force of the lubricant molecule.

The friction coefficients in the undiluted solutions of PEG200 to PEG600 in Fig. 8 were almost constant at sliding speeds of $0.5-15 \mathrm{~cm} / \mathrm{s}$. However, the friction coefficients in the undiluted solution of EG and the EG solutions containing PG400 and PEG1000 were about 0.3 which was twice as large as those in the solutions from PEG200 to PEG600. Because the friction force applied on the bottom point of the ball in the ball holder was measured by means of a transducer attached to the measuring arm in the high temperature tribometer, it would be related to the information of the lubricant film which was located at the specific heights above the stainless petri dish surface. The friction coefficients measured at the slow speed of $0.1 \mathrm{~cm} / \mathrm{s}$ and at the high speed of $15 \mathrm{~cm} / \mathrm{s}$ would correspond to the structure of the lubricating oil film formed on the petri dish surface and that in the bulk phase above the petri dish surface, respectively. Taking into account that the friction coefficients in the PEG600 solution were almost constant at the sliding speeds from $0.1-15 \mathrm{~cm} / \mathrm{s}$ in Fig. 8 , the structure of the lubricating oil film formed on the petri dish surface might be maintained in the bulk phase above the petri dish surface

The friction coefficients in the PEG200 and PEG400 solutions at sliding speeds of $0.5-15 \mathrm{~cm} / \mathrm{s}$ showed almost the same values as those in PEG600 at sliding speeds of $0.1-15 \mathrm{~cm} / \mathrm{s}$ in Fig. 8, although the PEG200 and PEG400 molecules on the petri dish surfaces formed the boundary lubricating film which was measured at a sliding speed of $0.1 \mathrm{~cm} / \mathrm{s}$. These facts indicate that the liquid lubricant structures just above the boundary lubricating film in the PEG200 and PEG400 solutions were similar to those in the PEG600 solution, although the chain lengths of the PEG200 and PEG400 molecules were different from those of the PEG600 molecules. Because the compressibility in Fig. 7 reflected the structure in the bulk phase of the lubricant, both the dependences of the friction coefficients and the compressibilities on the kinematic viscosities in the solutions from PEG200 to PEG600 should show the similar tendencies in Figs. 7 and 8. However, the frictional coefficient and the compressibility in the EG solution were different from those in the solutions from PEG200 to PEG600. These differences between EG and PEG seemed to depend on the presence of ether linkages -C-O-C- in the lubricant molecules. If the oxygen atoms of the ether linkages in the molecules from PEG200 to PEG600 were bound to the metal oxide surface, the friction coefficients in the solutions from PEG200 to PEG600 could be smaller than those in the EG solution.

\section{Conclusions}

The lubricant molecules in the water-glycol hydraulic fluid consisting of ethylene glycol and polyethylene glycol could be effectively cleaved by means of the intermittent ultrasonic irradiation at a frequency of $20 \mathrm{kHz}$ and output power of $40 \mathrm{~W}$ at 20 and $40{ }^{\circ} \mathrm{C}$. The kinematic viscosities in the lubricants decreased with the ultrasonic irradiation times because of the cleaving process of the lubricant molecules, while the ultrasonic velocities and compressibilities were almost constant. The friction coefficients and compressibilities in the undiluted solutions of polyethylene glycols with molecular weights from 200 to 600 were not be affected by the change in the kinematic viscosities except for those in ethylene glycol.

\section{Acknowledgement}

The author would like to thank Associate Professor David Richard Neill of Okayama University of Science for useful suggestions during this work.

\section{References}

[1] Lemar, R. L., "Factors Affecting Sonic Degradation of Polymer Solutions," Rock Island Arsenal, Rock Island, Ill., Tech. Rept., 6, 1, 1967, 19-23.

[2] Van Horne, W. L., "Viscosity Loss Effects of Polymeric Viscosity Modifiers," Division of Petroleum Chemistry, American Chemical 
Society, Preprints 1, No. 4, 1956, 26-31.

[3] Bowden, F. P. and Tabor, D., "The Friction and Lubrication of Solids," Clarendon Press, Oxford, 1986, 176.

[4] Jahanmir, S., "Chain Length Effects in Boundary Lubrication," Wear, 102, 1985, 331-349.

[5] Hachiya, K., "Vaporization and Oxidation of Poly- $\alpha$-olefin on Metal Plates," Studies Surface Sci. Catalysis, 132, 2001, 805-808.

[6] American Society for Testing Material, Philadelphia, Pa., "Supplementary Preprint to the 1961 Report of Committee D-2 on Petroleum Products and Lubricants," Appendix VII, 1961, 61-66.

[7] Nogami, S., "Shear Stability of Lubricating Oils and its Test Methods (1), On Ultrasonic Shear Stability Tests," JUNKATSU, 8, 2, 1963, 71-76.

[8] Czichos, H., "Tribology: A Systems Approach to the Science and Technology of Friction, Lubrication and Wear," Elsevier Scientific Publishing, 1978, 130.

[9] Lu, X., Khonsari, M. M. and Gelinck, E. R. M., "The Stribeck Curve: Experimental Results and Theoretical Prediction," ASME J. Tribol., 128, 2006, 789-794.

[10] McClements, D. J. and Powey, M. J. W., "Ultrasonic Solid Fat Content Determination,"
Ultrasonic International Conf. Proc., Butterworths, London, 1987, 43-47.

[11] McClements, D. J., Dickinson, E., Dungan, S. R., Kinsella, J. E., Ma, J. G. and Powey, M. J. W., "Effect of Emulsifier Type on the Crystallization Kinetics of Oil-in-Water Emulsions Containing a Mixture of Solid and Liquid Droplets," J. Colloid Interface Sci., 160, 1993, 293-297.

[12] Stuehr, J., Ultrasonic Methods, Techniques of Chemistry, Vol. 6, Part 2, ed. Hammes, G. G., Wiley-Interscience, NY, 1973, 237.

[13] Tanford, C., Physical Chemistry of Macromolecules, John Wiley \& Sons, NY, 1961, 390.

[14] Price, G. J., Ultrasonically Assisted Polymer Synthesis, Polymeric Materials Encylropedia, Vol. 11, ed. Salamone, J. C., CRC Press, Boca Raton, 1996, 8435.

[15] Wan, Y., Liu, W. and Xue, Q., "Effects of Diol Compounds on the Friction and Wear of Aluminum Alloy in a Lubricated Aluminum-on-Steel Contact," Wear, 193, 1996, 99-104.

[16] Okabe, H., Masuko, M. and Sakurai, K., "Dynamic Behavior of Surface-Adsorbed Molecules under Boundary Lubrication," ASLE Trans., 24, 1981, 467-473. 